\title{
Thermoelectric Properties of Heavy Fermion Compound $\mathrm{Ce}_{3} \mathrm{Co}_{4} \mathrm{Sn}_{13}$
}

\author{
P. Witas, L. Kalinowski, J. Goraus, M. FijaŁkowski and A. Ślebarski* \\ Institute of Physics, University of Silesia, Uniwersytecka 4, 40-007 Katowice, Poland
}

\begin{abstract}
The heavy fermion compound $\mathrm{Ce}_{3} \mathrm{Co}_{4} \mathrm{Sn}_{13}$ was studied in terms of its thermoelectric properties. To enhance its figure of merit we milled the solid sample and then pressed to obtain a consistent granulated material. The main properties, such as the Seebeck coefficient, thermal conductivity and electronic resistivity were measured at low $(<300 \mathrm{~K})$ temperatures for both, the solid and the granulated sample. Thermal conductivity was diminished and the Seebeck coefficient was slightly enhanced, while the resistivity of produced material was increased. We explain it by strong electron scattering on defects and grain boundaries present in the sample. The resulting figure of merit $Z T$ was found to be enhanced almost across the whole measured $T$ region.
\end{abstract}

DOI: 10.12693/APhysPolA.127.309

PACS: 71.27.+a, 72.15.Eb, 72.15.Jf

\section{Introduction}

In recent years, there has been a significant interest in the research of rare-earth based intermetallic compounds since they are potentially good thermoelectric materials. This type of compounds is often called heavy fermion systems due to exhibition of strong electronic correlations. The efficiency of a thermoelectric material is usually characterized by dimensionless figure of merit $Z T=S^{2} T /(\kappa \rho)$, where $S, \kappa$ and $\rho$ are the Seebeck coefficient (also referred to as thermopower), thermal conductivity and electrical resistivity, respectively. There are several main features of heavy fermions that considerably enhance their transport properties. Strong electronic correlation leads to the formation of narrow band near the Fermi level $E_{\mathrm{F}}$ with very high density of states $N(E)$ [1]. Since the Seebeck coefficient is proportional to the derivative $\mathrm{d} N(E) / \mathrm{d} E$ at $E_{\mathrm{F}}$, in heavy fermions it often reaches values of 10-100 times greater than those in ordinary metals [2]. While optimizing the $Z T$, it is desired to reduce the lattice thermal conductivity of a material. This involves boosting the phonon scattering. In this matter, especially promising are cage compounds, mainly clathrates and skutterudites. Filling these compounds with foreign atoms (rare earths) can significantly depress thermal conductivity due to "rattling" of host atoms inside the crystal lattice cages [3]. On the other hand, there is a different approach aimed at lowering the thermal conductivity. It involves preparation of material with finegrained structure by milling to a powder and then hot pressing. Dense distributed grain boundaries provides low phonon thermal conductivity. We report the $S, \rho$, $\kappa$ measurements and resulting $Z T$ of $\mathrm{Ce}_{3} \mathrm{Co}_{4} \mathrm{Sn}_{13}$ compound which crystallizes in a skutterudite-like structure and is reported to be a Kondo system [4]. Our aim is to compare the thermal and electronic transport of solid sample and its grained counterpart.

${ }^{*}$ corresponding author; e-mail: andrzej.slebarski@us.edu.pl

\section{Experimental}

Polycrystalline $\mathrm{Ce}_{3} \mathrm{Co}_{4} \mathrm{Sn}_{13}$ sample has been prepared by arc melting the constituent elements on a water cooled copper hearth in a high-purity argon atmosphere with an $\mathrm{Al}$ getter. The sample was remelted several times to promote homogeneity and annealed at $870^{\circ} \mathrm{C}$ for 12 days. Almost no mass loss $(<0.2 \%)$ occurred during the melting and annealing process. Sample was carefully examined by X-ray diffraction analysis and found to be a single phase with cubic structure (space group $P m \overline{3} n$ ). Part of the sample has been milled mechanically with agate mortar and pestle. Obtained powder has been characterized by taking an image with Zeiss optical microscope and grain size analysis using ImageJ software. Then, the powdered sample has been pressed under the pressure of $17 \mathrm{kbar}$. The almost identical stoichiometry of the solid and granulated samples was confirmed by the microprobe technique. In both samples the deviations from the nominal composition was less than $0.5 \%$.

All of the following thermoelectric measurements have been accomplished by a temperature gradient method $(S)$ and a four-contact method $(\kappa, \rho)$. The Physical Property Measurement System (PPMS) platform was used with Thermal Transport System (TTO) option. The measurement was carried out in the temperature range $5-300 \mathrm{~K}$.

\section{Results and discussion}

Figure 1 shows the microscopic image of milled $\mathrm{Ce}_{3} \mathrm{Co}_{4} \mathrm{Sn}_{13}$ powder. Black areas present the grains. From image analysis the granules were found to be in the range of $\approx 0.1-20 \mu \mathrm{m}^{2}$. Theoretical studies [5] suggest that thermoelectric properties of the Kondo systems should be highly sensitive to size of grains in the nanostructured material.

Our result of thermal conductivity measurement for $\mathrm{Ce}_{3} \mathrm{Co}_{4} \mathrm{Sn}_{13}$ solid sample presented in Fig. 2 is consistent with reported earlier data in [6]. As expected, the measured values $\kappa_{\text {exp }}$ are lower for pressed sample. The difference between $\kappa_{\exp }$ of solid and granulated sample is 


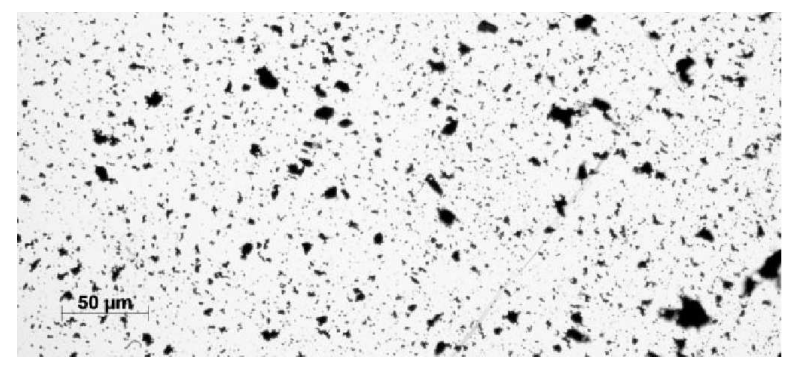

Fig. 1. Microscope image of milled $\mathrm{Ce}_{3} \mathrm{Co}_{4} \mathrm{Sn}_{13}$ powder dispersed in silicon oil.

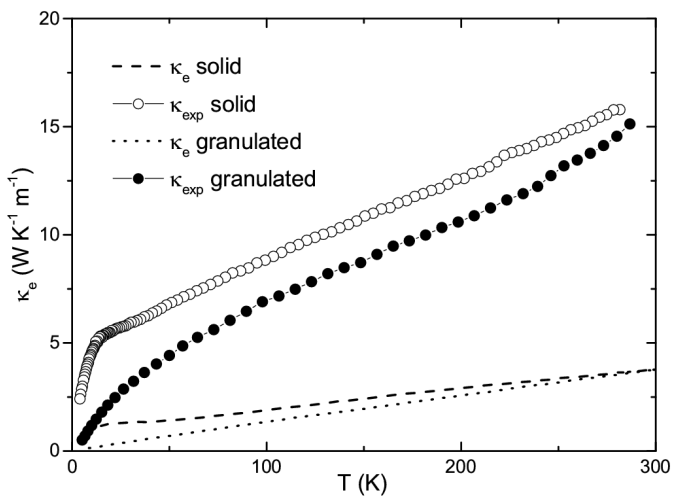

Fig. 2. Thermal conductivity of $\mathrm{Ce}_{3} \mathrm{Co}_{4} \mathrm{Sn}_{13}$. Comparison of pressed and solid sample. Dashed and dotted lines show estimated electronic contribution $\kappa_{\mathrm{e}}$.

nearly constant $\left(\approx 2 \mathrm{~W} \mathrm{~K}^{-1} \mathrm{~m}^{-1}\right)$ over the range 100 $240 \mathrm{~K}$. Near room temperature it begins to diminish and in low temperatures it grows up and has a maximum $\approx 3.5 \mathrm{~W} \mathrm{~K}^{-1} \mathrm{~m}^{-1}$ at $T=15 \mathrm{~K}$. In solid sample, the $\kappa(T)$ curve has a sharp hump at $13 \mathrm{~K}$, while in grained counterpart this hump is highly flattened.

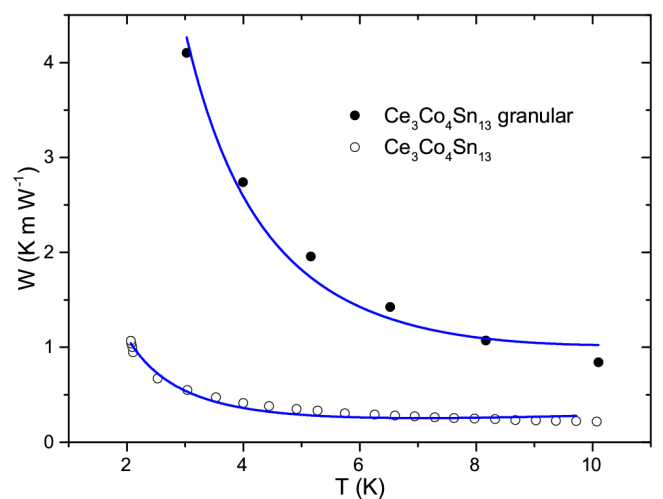

Fig. 3. Low temperature thermal resistivity of $\mathrm{Ce}_{3} \mathrm{Co}_{4} \mathrm{Sn}_{13}$ for granular and solid sample. Solid line represents theoretical fit with expression $W_{\mathrm{e}}=A T+\frac{B}{T^{2}}$, where $A=0.02 \mathrm{~mW}^{-1}, B=4.2 \mathrm{~K}^{3} \mathrm{~mW}^{-1}$ for solid and $A=0.06 \mathrm{~mW}^{-1}, B \stackrel{1}{=} 37.2 \mathrm{~K}^{3} \mathrm{~mW}^{-1}$ for granulated sample.
The thermal conductivity $\kappa$ of a metal consists of two components: an electronic $\kappa_{\mathrm{e}}$ and lattice $\kappa_{\mathrm{l}}$. The electronic contribution $\kappa_{\mathrm{e}}$ is directly related to the electronic conductivity of a material and it is described by the Wiedemann-Franz law $\kappa_{\mathrm{e}}=L_{0} T / \rho$, where $L_{0}$ is called the Sommerfeld value of the Lorenz number and its empirical value is $\pi / 3\left(k_{\mathrm{B}} / e\right)^{2}=2.44 \times 10^{-8} \mathrm{~W} \Omega \mathrm{K}^{-2}[7]$. The $\kappa_{\mathrm{e}}$ contribution vs. $T$ obtained for the both samples from the $\rho(T)$ data is shown in Fig. 2 .

The comparison of estimated $\kappa_{\mathrm{e}}$ and $\kappa_{\mathrm{exp}}$, indicates a small contribution of electronic thermal conductivity in the wide $T$-range. This conclusion is not valid at low temperatures, since the Wiedemann-Franz law is then not fulfilled [6].

Low temperature electronic thermal resistivity $W_{\mathrm{e}}$ is described by equation $W_{\mathrm{e}}=A T+\frac{B}{T^{2}}$ [7], where $A$ and $B$ are constants. The measured thermal resistivity $W=1 / \kappa_{\exp }$ fitted to the data and plotted for $T<10 \mathrm{~K}$ in Fig. 3 is well fitted with $W_{\mathrm{e}}$ (solid lines). The good fits suggest that the electronic thermal conductivity dominates within this temperature region.

In the paramagnetic region the resistivity $\rho(T)$ exhibits metallic conductivity $\rho(T)=\rho_{0}+\rho_{\mathrm{ph}}+\rho_{i}$ (Matthienssen's rule), where $\rho_{0}$ is residual resistivity and $\rho_{i}$ is an additional contribution to $\rho_{\mathrm{ph}}$ which represents electron-phonon scattering. In Fig. 4 the electrical

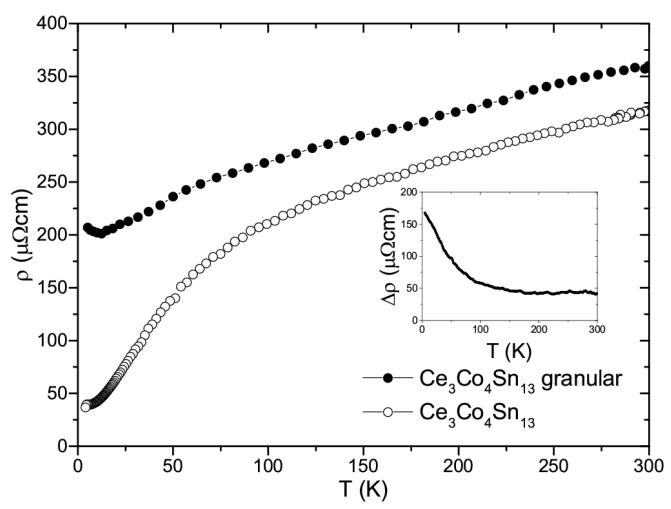

Fig. 4. Resistivity data for $\mathrm{Ce}_{3} \mathrm{Co}_{4} \mathrm{Sn}_{13}$ granular and solid sample. Inset shows resistivity difference $\Delta \rho$ of the respective samples.

resistivity $\rho(T)$ exhibits metallic conductivity, well approximated by the Bloch-Grüneisen-Mott formula

$$
\rho(T)=\rho_{0}+4 R T\left(\frac{T}{\Theta_{\mathrm{D}}}\right)^{4} \int \frac{x^{5} \mathrm{~d} x}{\left(\mathrm{e}^{x}-1\right)\left(1-\mathrm{e}^{-x}\right)}-K T^{3}
$$

( $\Theta_{\mathrm{D}}$ stands for the Debye temperature, $R$ and $K$ are constants, and $K T^{3}$ describes interband scattering process) [8] either for the solid or granular sample. Neglecting the crystal field effect and the possible spin fluctuations [4], the fits are good and give $\Theta_{\mathrm{D}}$ about $7 \mathrm{~K}$ larger for the granulated sample than $\Theta_{\mathrm{D}}$ of the solid one, which signals significant contribution of defects in the granulated sample. Assuming that $\rho_{0}$ is temperature independent, the different $\rho(T)$ behaviour for the granular $\mathrm{Ce}_{3} \mathrm{Co}_{4} \mathrm{Sn}_{13}$ can be related to the scattering 
of conduction electrons on grain boundaries and other structural defects. The linear $\Delta \rho$ vs. $T$ dependence displayed in the inset to Fig. 4 is characteristic of the strongly disordered alloys [9].

In Fig. 5 thermoelectric power $S(T)$ of the granular sample is $\approx 40 \%$ higher in relation to the solid one in the temperature region $150-200 \mathrm{~K}$. Moreover, the positive values of $S(T)$ suggest that the dominant charge carriers are holes, as has been reported in [6].

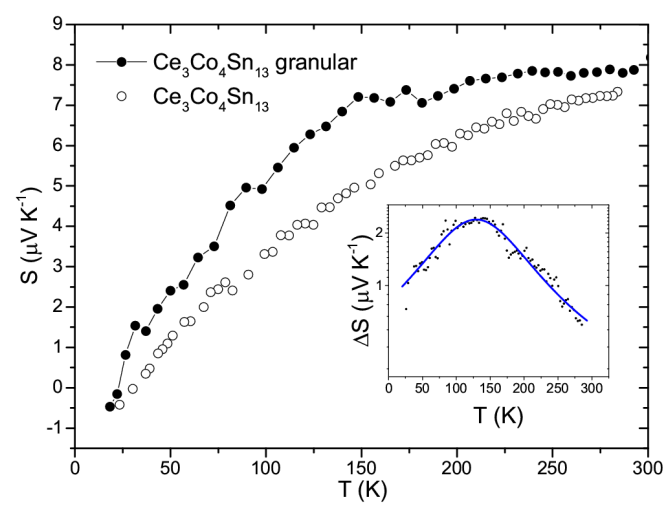

Fig. 5. Thermopower for $\mathrm{Ce}_{3} \mathrm{Co}_{4} \mathrm{Sn}_{13}$ granular and solid sample. Inset show difference $\Delta S=S_{\mathrm{G}}-S$ where $S_{\mathrm{G}}$ is granular sample thermopower.

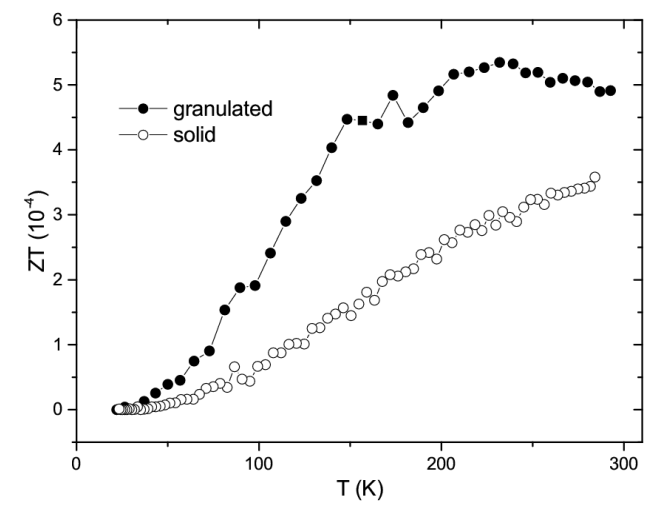

Fig. 6. Thermoelectric figure of merit for both $\mathrm{Ce}_{3} \mathrm{Co}_{4} \mathrm{Sn}_{13}$ samples.

Figure 6 shows temperature dependence of $Z T$. The aim of milling and pressing the sample was to enhance figure of merit and it was accomplished except the small low- $T$ region.

\section{Conclusion}

The granulation of heavy fermion cage type $\mathrm{Ce}_{3} \mathrm{Co}_{4} \mathrm{Sn}_{13}$ has positively changed the $Z T$. The enhancement of resistivity is compensated by increase of thermopower and reduction of thermal conductivity. Improvement of thermoelectric properties was accomplished by impact of scattering processes on grain boundaries and structural defects produced by milling and pressing. We have also shown that the leading contributions to the thermal conductivity have electrons at low temperatures and phonons at high temperatures.

\section{Acknowledgments}

We thank National Science Centre (NCN) for financial support, on the basis of decision No. DEC$2012 / 07 / \mathrm{B} / \mathrm{ST} 3 / 03027$. The research is also supported by the Forszt project co-financed by EU from the European Social Fund.

\section{References}

[1] J. Hubbard, Proc. R. Soc. Lond. A Math. Phys. Sci. 276, 238 (1963).

[2] R.J. Gambino, W.D. Grobman, A.M. Toxen, Appl. Phys. Lett. 22, 506 (1973).

[3] G.S. Nolas, J. Yang, H.J. Goldsmid, in: Thermal Conductivity Theory, Properties and Applications, Ed. T.M. Tritt, Kluwer-Academic, 2004.

[4] A. Ślebarski, B.D. White, M. Fijałkowski, J. Goraus, J.J. Hamlin, M.B. Maple, Phys. Rev. B 86, 205113 (2012).

[5] Y. Zhang, M.S. Dresselhaus, Y. Shi, Z. Ren, G. Chen, Nano Lett. 11, 1166 (2011).

[6] C.S. Lue, H.F. Liu, S.-L. Hsu, M.W. Chu, H.Y. Liao, Y.K. Kuo, Phys. Rev. B 85, 205120 (2012).

[7] J. Yang, in: Thermal Conductivity Theory, Properties and Applications, Ed. T.M. Tritt, Kluwer-Academic, 2004.

[8] N.F. Mott, H. Jones, The Theory of the Properties of Metals and Alloys, Oxford University Press, Oxford 1958.

[9] J.H. Mooij, Phys. Status Solidi A 17, 521 (1973). 